\title{
ANÁLISE DA VULNERABILIDADE À EROSÃO COSTEIRA ATRAVÉS DE GEOINDICADORES NAS PRAIAS DE PIEDADE E PAIVA (PE), BRASIL
}

\author{
COASTAL EROSION VULNERABILITY ANALYSIS THROUGH GEOINDICATORS IN PIEDADE \\ AND PAIVA BEACHES (PE), BRAZIL
}

\author{
Athos Farias MENEZES ${ }^{1}$, Pedro de Sousa PEREIRA ${ }^{1}$, Rodrigo Mikosz GONÇALVES ${ }^{1}$, \\ Tereza Cristina Medeiros de ARAÚJO ${ }^{1}$, Paulo Henrique Gomes de Oliveira SOUSA ${ }^{2}$ \\ ${ }^{1}$ Universidade Federal de Pernambuco (UFPE) - Centro de Tecnologia e Geociências - Recife - PE. \\ Emails: athos.farias1@gmail.com,psppraias@gmail.com,rodrigo.mikosz@gmail.com, terezaaraujo.ufpe@gmail.com \\ ${ }^{2}$ Universidade de São Paulo (USP) - Instituto Oceanográfico - São Paulo (SP) - Brasil. Email: sousaph@gmail.com

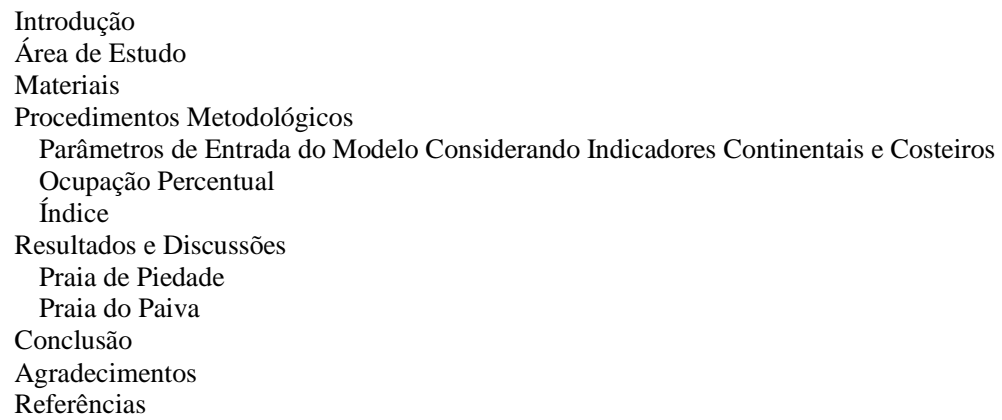

RESUMO - As praias de Piedade e Paiva estão localizadas no litoral do Estado de Pernambuco e pertencem aos municípios de Jaboatão dos Guararapes e Cabo de Santo Agostinho respectivamente. O presente trabalho tem como objetivo avaliar a vulnerabilidade à erosão costeira analisando dez geoindicadores com características continentais e costeiras. Como materiais para extrair tais informações foram utilizadas imagens de satélite de alta resolução e observações coletadas em campo. Os índices de vulnerabilidade foram classificados em três níveis de intensidade de acordo com a seguinte variação numérica e qualitativa: entre 0 a 2,9 (vulnerabilidade baixa), de 3 a 6,9 (vulnerabilidade moderada) e de 7 a 10 (vulnerabilidade alta). O setor norte e central da praia de Piedade foram os que obtiveram o maior valor do índice de vulnerabilidade à erosão costeira ambos iguais a 8,0 enquanto que 0 setor Sul da praia do Paiva apresentou um valor igual a 3,0. Destaca-se o uso desta modelagem para gestão costeira, sendo um procedimento considerado de baixo custo e que pode ser aplicado em diferentes escalas.

Palavras-chave: vulnerabilidade à erosão costeira, gestão integrada da zona costeira, sensoriamento remoto, sistema de informações geográficas (SIG).

\begin{abstract}
Piedade and Paiva beaches are located on the coast of Pernambuco state and belong to the municipalities of Jaboatão dos Guararapes and Cabo de Santo Agostinho respectively. This study aimed to assess the vulnerability to coastal erosion analyzing ten geoindicators with continental and coastal characteristics. As materials to extract this information were used high-resolution satellite images and in situ observation. The vulnerability index was classified into three levels of intensity in accordance with the following numerical and qualitative variation: from 0 to 2.9 (low vulnerability), 3 to 6.9 (moderate vulnerability) and 7 to 10 (high vulnerability). The northern and central sectors of Piedade beach was obtained the highest vulnerability to coastal erosion index equal to 8.0 for both, while the southern sectors of Paiva showed the lowest values equal to 3.0. It is highlighted the use of this modeling to support coastal management, being a low-cost procedure that could be applied at different scales.
\end{abstract}

Keywords: coastal erosion vulnerability, integrated coastal zone management, remote sensing, geographical information system (GIS).

\section{INTRODUÇÃO}

A zona costeira pode ser considerada como uma das áreas mais dinâmicas do planeta (Muehe, 2001). Seu monitoramento aplicado ao gerenciamento costeiro integrado torna-se necessário para mitigar impactos da erosão costeira que podem ser detectados e avaliados a partir de fatores naturais (fenômenos climáticos, geológicos e oceanográficos) e antrópicos (impermeabilização do solo, ocupação desordenada e retirada da vegetação natural) (Nascimento \& Dominguez, 2009; Silva et al., 2013). A vulnerabilidade à erosão costeira pode ser classificada através do uso de modelos analíticos considerando uma série de parâmetros passíveis de mensuração. Em vários lugares do mundo, encontram-se exemplos do grau considerado "alto" em uma determinada escala de classificação para vulnerabilidade, chegando a estágios críticos potencializados pelos fatores antrópicos, que normalmente ocasionam prejuízos ambientais, econômicos e sociais (Muehe, 2008; Neves \& Muehe, 2008; MMA, 2006).

Estudos de casos tais como trechos dos litorais espanhol (Doody, 2001), norte americano (Daniel, 2001; Morton et. al., 2004; Hapke et al., 2006; Hapke \& Plant, 2010), e brasileiro (Gonçalves et al., 2010; Silva et al., 
2013; Barreto et al., 2014; Nardez et al., 2016), são exemplos de trabalhos que buscam métodos capazes de classificar a vulnerabilidade à erosão costeira.

O conceito de vulnerabilidade costeira aproxima a susceptibilidade aos dados referentes à exposição, à capacidade de enfrentamento e aos sistemas físicos e sociais. Sendo muitas vezes visualizada como uma característica intrínseca de um sistema ou de um elemento, estando constantemente ampliado e abrangente a capacidade de adaptação do ambiente costeiro às mudanças antrópicas ocorridas (Birkmann, 2007).

Para a estimativa da vulnerabilidade à erosão costeira geoindicadores podem ser utilizados através de um conjunto de observações considerando parâmetros qualitativos e quantitativos. O principal objetivo deste tipo de modelagem é identificar e classificar o grau de intensidade (de baixo a alto) através de mapas temáticos ao longo de uma área, como visto em Harvey et al. (1999), Boruff et al. (2005), Mazzer et al. (2008); Mallman \& Araújo (2010); Raposeiro \& Ferreira (2010), Rudorff \& Bonetti (2010), Sousa et al. (2011) e Martins \& Pereira (2014).

Os geondicadores são utilizados para compreensão de cenários e podem ser aplicados para propor ações de monitoramento ambiental em escalas correspondentes às necessidades do estudo. Estes são integrados a bancos de dados que apresentam técnicas sofisticadas para avaliar informações geográficas com fins ambientais caracterizando-se como alternativas de baixo custo para a gestão costeira integrada no contexto público (Bush et al., 1999).

A maioria dos trabalhos que abordam monitoramento costeiro e vulnerabilidade à erosão costeira fazem uso de geotecnologias como fonte de informações geográficas utilizando sensores remotos (Gonçalves et al., 2010) tais como: fotografias aéreas a partir de aviões (Gonçalves et al., 2012a), RPA (Aeronaves Remotamente Pilotadas) e balões (Eulie et al., 2013), imagens de satélites artificiais (Malmann et al., 2014), videometria (Pereira et al., 2011; Pereira et al., 2012), informações de altimetria e modelos digitais do terreno utilizando LIDAR (Light Detection And Ranging) (Hantson et al., 2012), técnicas de posicionamento global como o GNSS (Global Navigation Satellite System) (Tanajura et al., 2011; Gonçalves et al., 2012b; Mendonça et al., 2014) e técnicas de levantamentos topográficos utilizando estação total (Gonçalves et al., 2013). Essas informações espaciais integradas passam a ser organizadas e analisadas de maneira quantitativa e qualitativa (Mazzer et al., 2008).

A partir de um estudo de caso no nordeste do Brasil, mais especificamente nas praias de Piedade e do Paiva localizadas no estado de Pernambuco, este trabalho apresenta uma contribuição para a análise dos geoindicadores continentais e costeiros, destacando-se os seguintes objetivos: (i) avaliar a vulnerabilidade à erosão costeira na área de estudo aplicado a metodologia proposta por Sousa et al. (2013). (ii) fazer uso de observações obtidas por sensoriamento remoto orbital e desta forma extrair informações e parâmetros necessários ao cálculo da vulnerabilidade (iii) fornecer através da classificação encontrada subsídios para o planejamento e gerenciamento dos recursos costeiros.

\section{ÁREA DE ESTUDO}

A área de estudo diz respeito as duas praias pertencentes ao litoral do Estado de Pernambuco, sendo elas a praia do Paiva (Cabo de Santo Agostinho) e a praia de Piedade (Jaboatão dos Guararapes). A figura 1 está dividida da seguinte forma: (a) a localização de Pernambuco no Nordeste do Brasil, (b) os municípios em estudo e (c) uma imagem de satélite com a localização mais detalha das praias em questão.

A praia do Paiva localiza-se ao norte do município do Cabo de Santo Agostinho, seus limites geográficos são com o Rio Jaboatão ao Norte e com a praia de Itapuama ao Sul. O litoral
Norte do município do Cabo de Santo Agostinho vem passando por um processo de urbanização, como calçadões, equipamentos esportivos e de lazer, pistas de rolamento, calçadas, jardins e prédios que caracterizam a perspectiva de ambiente natural devido a influência antrópica (Manso et al., 2006).

De maneira natural, a vegetação da praia do Paiva é identificada como mata atlântica e áreas de mangue, que ao longo da história passou por um processo de gradativa mudança através da substituição da flora natural para coqueiros e até mesmo a própria urbanização sob influência das 
ações logísticas do estado.

A praia de Piedade está localizada no município de Jaboatão dos Guararapes-PE, na porção central da região Metropolitana do Recife (Fig.1 (a) e (c)). Possui características diferentes da praia do Paiva nos aspectos físicos devido a existência de duas linhas de arrecife, protegendo a praia da incidência de ondas. Diferencia-se também pelos aspectos socioeconômicos sendo uma praia completamente urbanizada e obtendo uma densidade demográfica considerada alta com 2.491,82 hab $/ \mathrm{km}^{2}$ (IBGE, 2010).
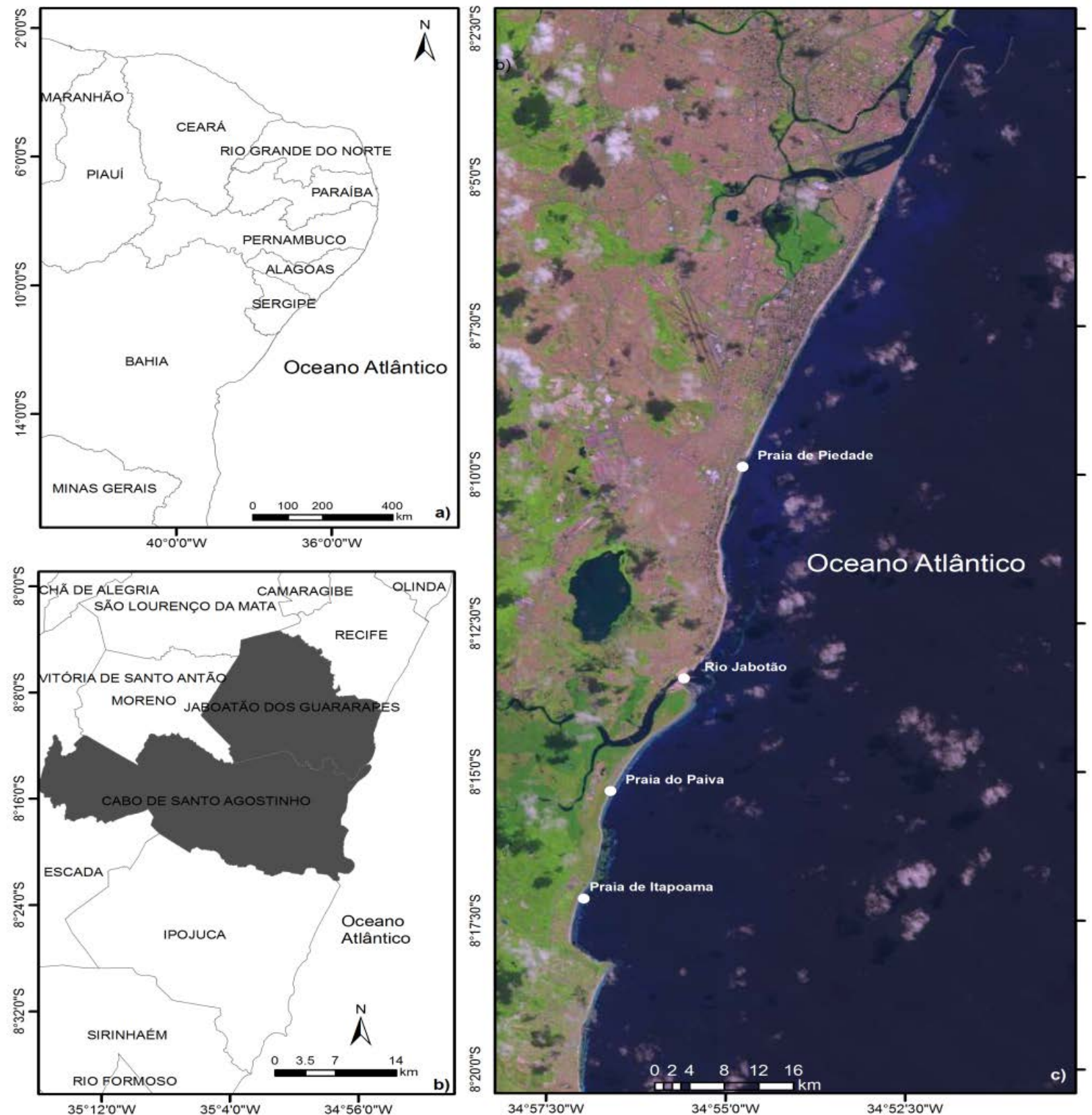

Figura 1 - a) Região Nordeste; b) Municípios de Jaboatão dos Guararapes e Cabo de Santo Agostinho no Estado de Pernambuco; c) Imagem de satélite indicando as praias do Paiva e Piedade. Datum SIRGAS 2000. Sistemas de Coordenadas Geográficas.

Cada setor foi dividido através de suas peculiaridades ambientais e/ou intervenções antrópicas. O setor norte foi caracterizado pela vegetação densa em uma área próxima à costa além da foz do Rio Jaboatão. No setor central é encontrada uma dinâmica residencial de alguns condomínios de alto padrão. E o setor sul foi destacado pela presença de um Recife de Arenito com presença coralínea em sua superfície (Dominguez et al., 1990).

Assim como a praia do Paiva, a praia de Piedade também foi dividida em 3 setores. O setor norte é caracterizado pela ausência de recifes e estruturas costeiras, o central pela presença de recife e o sul apresentou, além de recifes, uma estrutura de engenharia costeira. 


\section{MATERIAIS}

Com o objetivo de realizar o mapeamento das áreas de interesse foram utilizadas imagens orbitais de alta resolução espacial.

As imagens digitais utilizadas são do satélite artificial Geoeye do ano de 2013, com as seguintes características de resolução espectral por bandas: azul (450-510nm); verde (510$580 \mathrm{~nm})$; vermelho (655-690nm); infravermelho próximo (780-920nm) e pancromática (450$800 \mathrm{~nm}$ ) com resolução espacial para o modo pancromático de 0,41 m e 1,65 m para as demais bandas espectrais. Estas foram cedidas pelo LACCOST - Laboratório de Cartografia Costeira da UFPE e foram adquiridas através do projeto FACEPE/Processo: PPP/APQ-1242-1.07/10).

A partir destas imagens foram extraídas informações espaciais dos geoindicadores, inseridas em um banco de dados geográficos e utilizadas para a análise estatística e parâmetro de entrada do método proposto.

\section{PROCEDIMENTOS METODOLÓGICOS}

\section{Parâmetros de entrada do Modelo considerando Indicadores Continentais e Costeiros}

Com o intuito de minimizar os impactos da erosão costeira, Bush et al. (1999) apresentam um simples e rápido método baseado em indicadores ambientais e geoindicadores. A tabela 1 apresenta três principais colunas do índice (baixo, moderado e alto), duas variáveis (costeiras e continentais) e dez indicadores (morfologia da praia, posição da linha de costa, presença de dunas, exposição às ondas, presença de rios, elevação do terreno, vegetação, estruturas costeiras, ocupação percentual e solo permeabilizado).

Este método aborda variáveis que influenciam na vulnerabilidade à erosão costeira, a partir da análise de geoindicadores naturais e antrópicos (Sousa et al., 2011). A seguir é apresentada a descrição de cada geoindicador:

a) Morfologia da praia: Este é o primeiro e mais importante geoindicador para compreender os processos dinâmicos da linha de costa. A morfologia costeira é o fator resultante de processos hidrodinâmicos, geológicos e climatológicos. Podendo ocorrer em longa ou curta duração, na ordem de metros a quilômetros, respectivamente (Menezes, 2008).

b) Posição da linha de costa: Este elemento sofre constantes alterações, sendo que estas podem ser intensas ou pouco significativas, não possuindo um padrão indicado em escalas temporais. Para avaliar este indicador é necessário avaliar as alterações históricas do ambiente praial como indicam Cowel \& Thom (1994).

c) Campos de dunas: Funcionam como barreiras naturais que protegem a zona costeira de ondas e tempestades, além de funcionarem como fontes de sedimentos para as praias adjacentes. Elas têm uma importância elementar, pois são feições eficazes na proteção praial.

d) Recifes: Este é um parâmetro que define o grau de exposição ou proteção da linha de costa para a ação de ondas. É necessário observar a existência ou ausência de obstáculos em direção ao longo da linha de costa.

e) Rios ou canais: Os rios e canais são feições que tendem à instabilidade em função de sua migração ao longo do tempo. Rios e canais podem migrar muitos metros causando mudanças abruptas na linha de costa.

f) Elevação do terreno: Está relacionada com o alcance do ataque das ondas e elevação do nível do mar. Uma região com elevação acima de $6 \mathrm{~m}$ dificilmente sofrerá inundações causadas por sobrelavagem que ocorrem em períodos energéticos. Já terrenos com elevação abaixo de $3 \mathrm{~m}$ são bastante suscetíveis à ação destes eventos.

g) Vegetação: A vegetação na zona costeira é um indicador que mostra estabilidade ou instabilidade de uma área adjacente à praia. A vegetação funciona como retentora de sedimentos. Além disso, quando arbórea com copas altas e bem desenvolvidas, indica estabilidade de uma área com processo de pedogênese avançada e ausência de indícios de erosão costeira, enquanto que a vegetação com raízes expostas e árvores inclinadas sugere processo de erosão em curso.

h) Engenharia costeira e estruturas de contenção: As estruturas de engenharia costeira são comumente implantadas para tentar minimizar ou conter os impactos da erosão. Estas obras são implantadas em momentos de 
emergências para manter a infraestrutura urbana localizada nas proximidades da linha de costa. Embora providencie a proteção em curto prazo, elas representam áreas de instabilidade em cenário futuro.

i) Ocupação: uma maior densidade populacional na zona costeira aumenta a vulnerabilidade, pois os impactos da erosão podem gerar prejuízos socioeconômicos significativos.

A ocupação pode intensificar processos erosivos ao intervir no balanço sedimentar da praia.

j) Impermeabilidade do solo: Este indicador está diretamente relacionado ao percentual de ocupação. Quanto maior a ocupação, maior é a impermeabilidade do solo em função da infraestrutura urbana como residências e vias.

Tabela 1 - Geoindicadores utilizados para avaliação da vulnerabilidade à erosão costeira (Sousa et al., 2011).

\begin{tabular}{|c|c|c|c|c|c|}
\hline \multirow{11}{*}{ 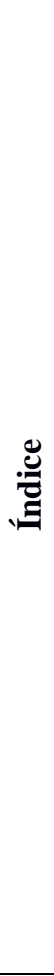 } & Variáveis & Indicadores & V. Baixa & V. Moderada & V. Alta \\
\hline & \multirow{5}{*}{$\frac{\mathscr{g}}{\tilde{y}}$} & $\begin{array}{l}1 \text { Morfologia da } \\
\text { praia }\end{array}$ & $\begin{array}{l}\text { Bom suprimento } \\
\text { sedimentar. }\end{array}$ & Praia estreita & Ausência de praia \\
\hline & & $\begin{array}{l}2 \text { Posição da linha } \\
\text { de costa }\end{array}$ & Acresção & Estável & Erosão \\
\hline & & 3 Campos de Dunas & $\begin{array}{l}\text { Grande extensão } \\
\text { de campos de } \\
\text { dunas. }\end{array}$ & $\begin{array}{l}\text { Dunas de baixas } \\
\text { altitudes. }\end{array}$ & Sem dunas \\
\hline & & 4 Exposição às ondas & $\begin{array}{l}\text { Presença de } \\
\text { barreiras naturais. }\end{array}$ & $\begin{array}{l}\text { Presença de } \\
\text { bancos de areia. }\end{array}$ & Ondas sem obstáculos naturais \\
\hline & & $\begin{array}{l}5 \text { Distância da foz de } \\
\text { Rios }\end{array}$ & $>100 \mathrm{~m}$ & $\begin{array}{l}\text { Entre } 50 \text { e } 100 \\
\mathrm{~m}\end{array}$ & $<50 \mathrm{~m}$ \\
\hline & \multirow{5}{*}{ نُ } & $\begin{array}{l}6 \text { Elevação do } \\
\text { terreno }\end{array}$ & $>6 \mathrm{~m}$ & 3-6 m & $<3 \mathrm{~m}$ \\
\hline & & 7 Vegetação & Vegetação Densa & $\begin{array}{l}\text { Gramíneas, } \\
\text { Arbustos }\end{array}$ & Ausência de Vegetação \\
\hline & & 8 Estruturas costeiras & Ausente & $\begin{array}{l}\text { Estruturas de } \\
\text { pequeno porte }\end{array}$ & $\begin{array}{l}\text { Muros de contenção ao longo do } \\
\text { ambiente praial. }\end{array}$ \\
\hline & & $\begin{array}{l}9 \text { Percentual de } \\
\text { ocupação do solo }\end{array}$ & $<30 \%$ & Entre 30 e $70 \%$ & $>70 \%$ \\
\hline & & $\begin{array}{l}10 \text { Impermeabilidade } \\
\text { do solo }\end{array}$ & $\begin{array}{l}\text { Ausência de } \\
\text { ocupações }\end{array}$ & $\begin{array}{l}\text { Baixo } \\
\text { quantitativo de } \\
\text { ocupações }\end{array}$ & $\begin{array}{l}\text { Obras de infraestrutura avançadas: } \\
\text { assentamentos desenvolvidos, } \\
\text { estradas asfaltadas e urbanização } \\
\text { elevada. }\end{array}$ \\
\hline
\end{tabular}

\section{Ocupação Percentual}

Para a análise da vulnerabilidade à erosão costeira nas duas praias avaliadas foram necessários estudos acerca da densidade demográfica e ocupação urbana, que foram obtidas através dos dados populacionais do IBGE (2010) e análise do cenário encontrado nas imagens de satélite respectivamente.

O índice de ocupação $\left(A_{o}\right)$ é a soma de todas as variáveis e feições urbanas como descrito na equação 1:

$$
A_{0}=A_{b}+A_{r}+\ldots+A_{n}
$$

Onde $A_{b}$ são áreas edificadas; $A_{r}$ são áreas de estradas e $A_{n}$ são $n$ variáveis. A ocupação percentual é definida como $\left(P_{o}\right)$ (Equação 2):

$$
P_{o}=\frac{A_{o} \times 100}{A_{u}}
$$

- onde $A_{u}$ corresponde ao total da área em cada setor. Porcentagens inferiores a 30\% indicam baixa vulnerabilidade, e porcentagens superiores $70 \%$ indicam a vulnerabilidade alta. Valores intermediários indicam vulnerabilidade moderada.

\section{Índice}

Segundo Bush et al. (1999) e Sousa et al. (2011) os setores dos ambientes costeiros são resultados dos processos físicos e antrópicos integrados, através de agentes dinâmicos que promovem alterações contínuas no ambiente. Deste modo, o índice avalia cada indicador de maneira individual conforme descrito no item anterior.

O indicador é classificado conforme seu potencial para a vulnerabilidade à erosão costeira (baixa, moderada ou alta), considerando os valores da tabela 1 . Um valor numérico baseado nas amostras é atribuído para 
cada variável: $0=$ baixa, $5=$ moderada e 10 para alta vulnerabilidade. Os mesmos são organizados em planilhas que alimentam a base de dados para o modelo numérico.

Os resultados obtidos através do banco de dados acerca da vulnerabilidade costeira são aplicados na equação 3 , que resulta no índice (I), de acordo com os 10 parâmetros de entrada do modelo. De acordo com a equação 3 temos:

$$
I=\left(\frac{1}{n_{v}}\right) \cdot \sum_{v}\left(\frac{1}{n_{i}}\right) \cdot \sum_{i} x_{i}
$$

Onde $n_{v}$ é o número de variáveis, $n_{i}$ o número de indicadores de determinada variável e $x_{i}$ é a soma dos indicadores.

Somando as variáveis e realizando a média aritmética dos indicadores, obtém-se o valor do índice proposto.

A vantagem deste índice é a possibilidade de trabalhar em diferentes escalas de mapeamento com base na disponibilidade de dados de entrada para a área de interesse.

O índice é um resumo dos indicadores (Tabela 1), dada pela avaliação da vulnerabilidade sendo os valores numéricos encontrados entre 0 a 2,9 considerado como baixa vulnerabilidade, de 3 a 6,9 vulnerabilidade moderada e de 7 a 10 como vulnerabilidade alta.

\section{RESULTADOS E DISCUSSÕES}

Para uma melhor descrição e entendimento dos resultados acerca do índice de vulnerabilidade à erosão costeira, cada praia é apresentada e qualificada a partir dos setores como observado abaixo.

\section{Praia de Piedade}

A praia de Piedade passa por um intenso processo de urbanização devido à especulação imobiliária.

Ao longo de sua orla é possível observar diversos empreendimentos de grande porte, sendo a maioria de finalidade residencial. Esta perspectiva vem sendo observada como uma tendência ao longo da porção do núcleo metropolitano (Costa et. al, 2008).

Todos os setores da praia de Piedade obtiveram valores maiores ou iguais a 7 pontos (Setor Norte $=8,0$; Setor Central $=8,0$; e Setor Sul $=7,0$ ), sendo assim contabilizados como altos valores no índice de vulnerabilidade à erosão costeira. O único indicador que apresentou valores de baixa vulnerabilidade para todos os setores foi a distância para foz de rios, este elemento não foi observado na imagem de satélite utilizada para o estudo. Os valores encontrados estão disponíveis na tabela 2 .

Tabela 2 - Resultados da avaliação da vulnerabilidade à erosão costeira por setores na Praia de Piedade.

\begin{tabular}{l|c|c|c}
\hline Indicadores & Setor Norte & $\begin{array}{c}\text { Setor } \\
\text { Central }\end{array}$ & Setor Sul \\
\hline Morfologia da praia & 5 & 5 & 5 \\
\hline Posição da linha & 10 & 10 & 10 \\
\hline Campos de Dunas & 10 & 10 & 10 \\
\hline Exposição às ondas & 10 & 5 & 0 \\
\hline Distância da foz de Rios & 0 & 0 & 0 \\
\hline Elevação do terreno & 5 & 5 & 5 \\
\hline Vegetação & 5 & 10 & 10 \\
\hline Estruturas costeiras & 10 & 10 & 10 \\
\hline Percentual de ocupação & 10 & 10 & 10 \\
\hline Impermeabilidade do solo & 10 & 10 & 10 \\
\hline Total & 7,5 & 7,5 & 7,0 \\
\hline
\end{tabular}

A importância econômica da localização da praia de Piedade para a Região Metropolitana do Recife reflete a alta urbanização nos três setores, onde foram encontrados $57,8 \%$ de ocupação antrópica para o setor norte, 60,9\% para o setor central e $56,4 \%$ para o setor sul.
De acordo com Guerra \& Manso (2004) o aumento das edificações e estradas ao longo da região de pós-praia é fator condicionante à potencialização do balanço sedimentar negativo que, quando associado a processos erosivos, contribui para o recuo da linha de costa na região. 
Ainda com referência às variáveis costeiras, a análise da vegetação para os três setores foi considerada muito baixa, pois ao longo da orla na praia de Piedade são observados alguns poucos arbustos no setor Norte e Sul, já no setor Central não existe vegetação no ambiente praial. A figura 2 apresenta os resultados da classificação do uso do solo para a praia de Piedade. Até meados de 2013, os setores central e sul apresentavam grandes obras de engenharia para conter a erosão costeira. Após o processo de engorda da costa de Jaboatão dos Guararapes, concluído em 2014, a estrutura do setor Central foi retirada e a do setor Sul foi reduzida. Este processo caracterizou na inserção de 1 milhão de $\mathrm{m}^{3}$ de areia ao longo de $5,6 \mathrm{~km}^{2}$ do litoral de Jaboatão dos Guararapes. A Jazida destes sedimentos estava localizada nas proximidades da praia de Calhetas, no município do Cabo de Santo Agostinho (CPRH, 2014).

Após a engorda da praia a proposta é que haja uma manutenção em 5 anos. Entretanto, a ideia de fixação da linha de costa com este projeto é de que ela permaneça estável e ocorram apenas reparos nas áreas onde houver erosão (CPRH, 2014).

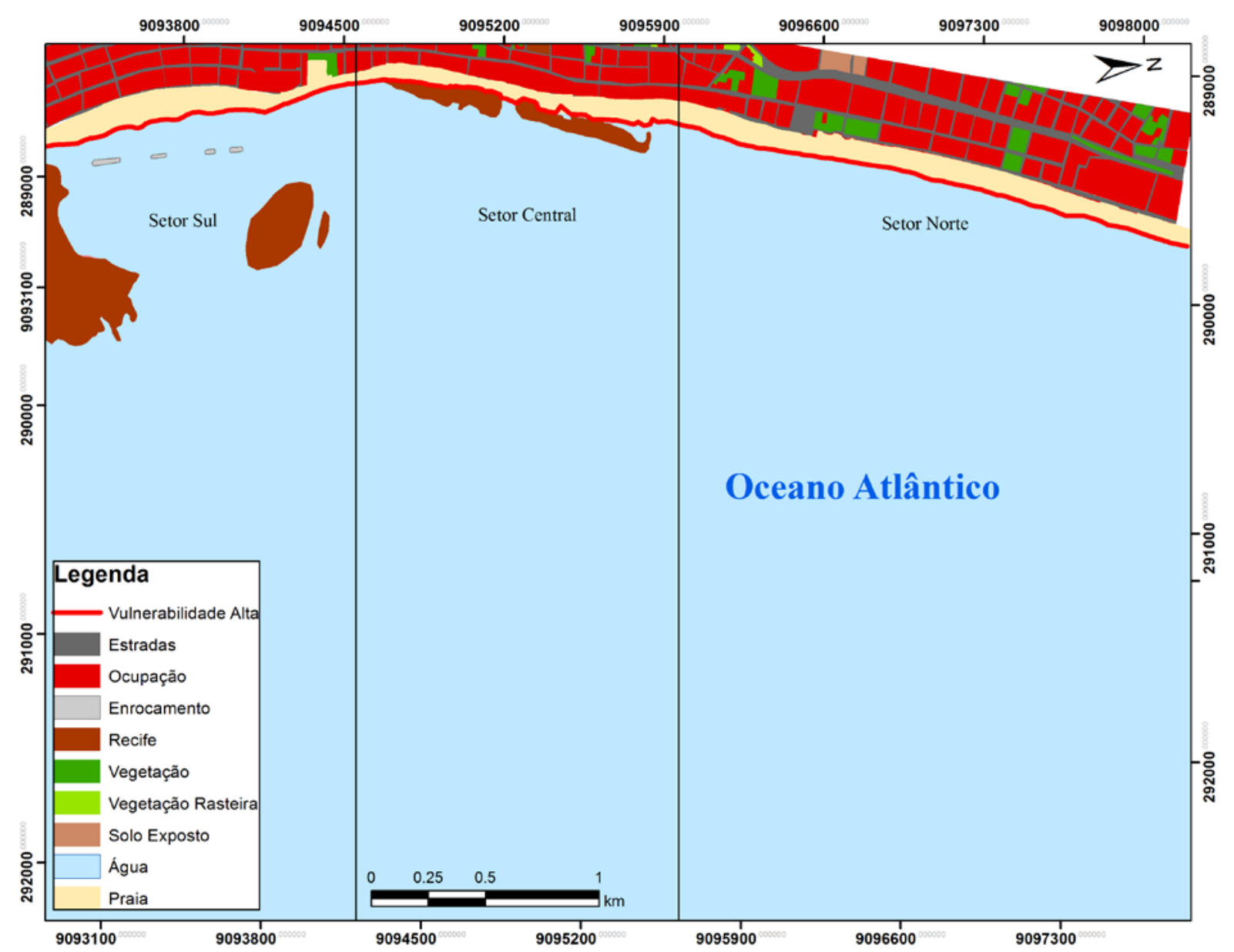

Figura 2 - Síntese dos resultados da classificação do uso do solo na Praia de Piedade, PE.

A distância média da praia de Piedade está entre 100 e 150 m entre a linha d'água e a orla urbanizada. Estes valores são verificados ao longo da imagem de satélite obtida anteriormente ao processo de engorda. Destacase que em alguns locais antes da engorda era observado, durante a maré alta, o contato direto do mar com o perímetro urbano (Manso et al., 2012). Mesmo após o processo de engorda, realizado pela prefeitura de Jaboatão dos Guararapes, a praia de Piedade apresenta um perfil topográfico entre $3 \mathrm{~m}$ e $6 \mathrm{~m}$ de altura. $\mathrm{O}$ entendimento da morfologia costeira para a praia de Piedade é um parâmetro importante para entender os mecanismos que dominam a dinâmica sedimentar e os subsequentes processos erosivos que ocorreram na região. Ao longo de todo o perfil praial, na praia de Piedade, foi observado a ausência de campos de dunas.

De acordo com a metodologia de Bush et al., (1999) esta característica representa um fator 
que pode potencializar os processos erosivos. No setor norte da presente praia não são observados recifes, entretanto, para os setores central e sul verifica-se a presença destes. Os mesmos são classificados como recifes de arenito segundo Dominguez (1990). Neste caso, a exposição às ondas foi considerada como um geoindicador classificado como alto para o setor norte, o setor sul não está exposto às ondas (valores baixos), enquanto o setor central obteve valores médios.

\section{Praia do Paiva}

$\mathrm{Na}$ praia do Paiva destacam-se algumas variáveis em destaque, como a presença expressiva de vegetação de coqueiros, remanescentes da Mata Atlântica secundária e manguezais, estes últimos, de acordo com Sacramento et al. (2007), identificados como Conocarpus erectus.

Os resultados encontrados indicam vulnerabilidade à erosão costeira média e muito semelhante para todos os setores conforme os seguintes valores numéricos encontrados: Norte $=4,5$; Central $=4,5$; $\mathrm{Sul}=$ 3,0. A tabela 3 apresenta os resultados para a praia do Paiva.

Tabela 3 - Resultado da avaliação de vulnerabilidade total e por setor da Praia do Paiva.

\begin{tabular}{l|c|c|c}
\hline \multicolumn{1}{c|}{ Indicadores } & Setor Norte & Setor Central & Setor Sul \\
\hline Morfologia da praia & 0 & 5 & 5 \\
\hline Posição da linha & 5 & 5 & 5 \\
\hline Campos de Dunas & 10 & 10 & 10 \\
\hline Exposição às ondas & 10 & 10 & 5 \\
\hline Distância da foz de Rios & 10 & 0 & 0 \\
\hline Elevação do terreno & 5 & 5 & 5 \\
\hline Vegetação & 0 & 0 & 0 \\
\hline Estruturas costeiras & 0 & 0 & 0 \\
\hline Percentual de ocupação & 5 & 5 & 0 \\
\hline Impermeabilidade do solo & 0 & 5 & 0 \\
\hline Índice & 4,5 & 4,5 & 3,0 \\
\hline
\end{tabular}

Os valores percentuais para os setores Norte, Sul e Central para a área vegetada estiveram contabilizados acima de 55\%, com o destaque para o setor sul, onde o valor percentual para vegetação foi de $57,8 \%$.

A perspectiva da presença de vegetação ao longo da praia do Paiva se mostra bastante evidente que pode ser observada no mapa de uso do solo na figura 3. Em contrapartida, o aspecto urbano segue com a presença de residenciais de luxo nos ambientes próximos à faixa de praia (Manso, 2012).

Os setores que apresentam modificações do ambiente natural impermeabilizando o solo são os setores Norte, devido à proximidade com o município de Jaboatão dos Guararapes, e o setor Central com a presença dos novos residenciais que se referem a especulação imobiliária local (Madruga Filho \& Araújo, 2000). O uso e ocupação do solo nos três setores da praia do Paiva não ultrapassaram os valores de 25,9\%.

Entretanto, este setor já apresenta alterações com a criação de condomínios de luxo, considerando a presença local da especulação imobiliária para a construção de novos empreendimentos, como já supracitado.

Os setores Norte e Sul contabilizaram valores de urbanização de $10,4 \%$ e $22,7 \%$, respectivamente.

Neste contexto é possível observar que se trata de uma praia com características de preservação, ou seja, o ambiente natural pouco alterado.

Além deste aspecto o aporte sedimentar é algo a ser destacado, sendo o pontal do Paiva o trajeto de maior largura de praias neste estudo, com $445 \mathrm{~m}$. Apesar disto, a faixa de praia, ao longo dos três setores, não ultrapassa $150 \mathrm{~m}$ de extensão.

Quanto ao aspecto topográfico, de acordo com Malmann et al., (2014) a praia do Paiva não possui um perfil topográfico superior a $6 \mathrm{~m}$ de altura o que propicia uma maior vulnerabilidade para o local. Semelhante à praia de Piedade, a Praia do Paiva não apresenta 
Dunas ao longo do seu perfil. Com relação à presença de recifes ao longo da costa da praia do Paiva apenas o setor Sul conta com a presença de um grande recife próximo a linha de costa.

De acordo com Dominguez (1990), trata-se de um recife de arenito. Diferentemente do setor Sul, os setores Central e Norte são expostos à ação das ondas.

Por tratar-se de uma praia com pouca interferência antrópica, não há estruturas costeiras.

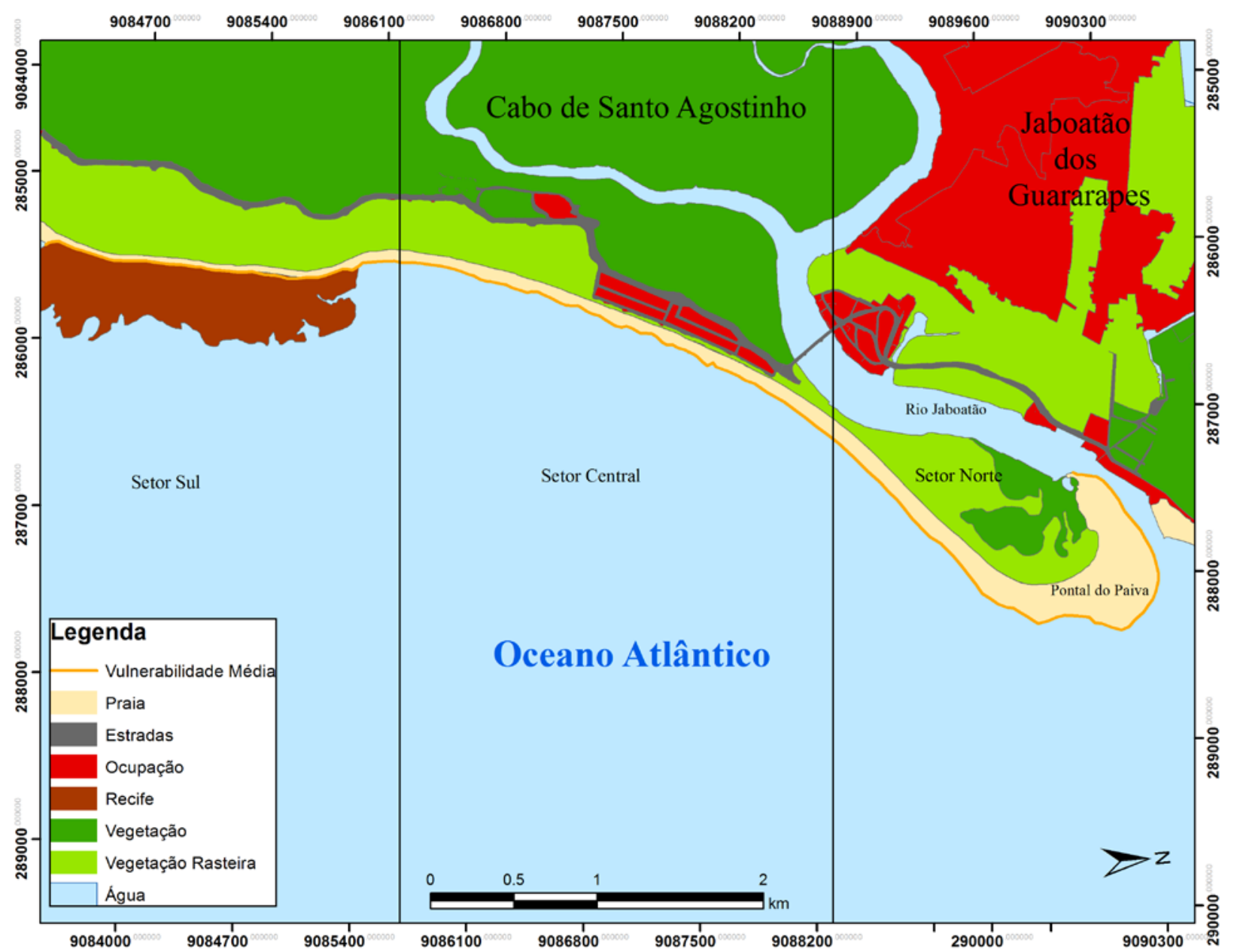

Figura 3 - Síntese da avaliação de vulnerabilidade da Praia do Paiva, PE.

\section{CONCLUSÕES}

Foi realizada uma avaliação da vulnerabilidade à erosão costeira nas praias de Piedade e do Paiva em Pernambuco. Após análise dos dez geoindicadores para as praias estudadas, verificou-se que ambas apresentaram vulnerabilidades diferentes variando de média a alta. Entre os resultados encontrados foi possível observar intensas áreas de urbanização nos três setores da praia de Piedade que contrastam com cenários de áreas de manguezais e vegetação nos setores central e sul da praia do Paiva.

O presente método pode ser adequado para outras áreas modificando as variáveis de entrada que representam os geoindicadores conforme a disponibilidade e acesso a informações, até mesmo acrescentando novas informações ao modelo. Como sugestão de novos geoindicadores para trabalhos futuros poderiam ser incluídos características e informações a respeito do regime de maré, ondas, perfil topográfico, batimetria, entre outros.

Outra questão, que ainda pode ser explorada é a inserção de pesos específicos para determinados geoindicadores. Neste estudo, todos os indicadores receberam o mesmo peso.

Os resultados encontrados neste trabalho podem ser utilizados como suporte para tomada de decisão visando detectar e reduzir os prejuízos causados pela erosão costeira, bem como suporte para manutenção, preservação e planejamento da infraestrutura urbana. Isto reforça a importância de avaliar a vulnerabilidade à erosão costeira através de geoindicadores, bem como dar continuidade ao monitoramento costeiro. 


\section{AGRADECIMENTOS}

Os autores agradecem à FACEPE (Fundação de Amparo à Ciência e Tecnologia de Pernambuco) pela bolsa de Mestrado de A. F. Menezes e pela disponibilidade das imagens de satélite utilizadas para este trabalho adquiridas pelo Processo: PPP/APQ-1242-1.07/10. À FACEPE /FAPESP (Vulnerabilidade da zona costeira dos estados de São Paulo e Pernambuco: situação atual e projeções para cenários de mudanças climáticas - APQ 0068-1.08/09 e $n^{0}$. 09/52564-0) e ao Processo no 310412/2015 - 3/PQ/nível 2/CNPq.

\section{REFERÊNCIAS}

AMARO, V.E.; SANTOS, M.S.T.; SOUTO, M.V.S. Geotecnologias Aplicadas ao Monitoramento Costeiro: Sensoriamento Remoto e Geodésia de Precisão. $1^{\text {a }}$ Ed. Natal, Edição dos Autores, 118p. 2012.

BARRETO, A.M.F.; SUGUIO, K.; BEZERRA, F.H.R.; TATUMI, S.H.; YEE, M.; GIANNINI, P.C.F. Geologia e geomorfologia do Quaternário costeiro do Rio Grande do Norte. Geologia USP. Série Científica, v. 4, n. 2, p. 1-12, 2004.

BIRKMANN, J. Risk and vulnerability indicators at different scales: applicability, usefulness and policy implications. Environmental Hazards, v. 7, p. 20-31, 2007.

BORUFF, B.J.; EMRICH, C.; CUTTER, S.L. Erosion hazard vulnerability of US coastal counties. Journal of Coastal Research, v. 21, n. 5, p. 932-942, 2005.

BUSH, D.M.; NEAL, W.J.; YOUNG, R.S.; PILKEY, O.H. Utilization of geoindicators for rapid assessment of coastalhazard risk and mitigation. Ocean \& Coastal Management, v. 42, n. 8, p. 647-670, 1999.

COOPER, J.A.G. \& MCLAUGHLIN, S. Contemporary multidisciplinary approaches to coastal classification and environmental risk analysis. Journal of Coastal Research, v. 14, n. 2, p. 512-524, 1998.

COWEL, P.J. \& THOM, B.G. Morphodynamics of coastal evolution. In: CARTER, R.W.G. \& WOODROFFE, C.D. (eds), Coastal Evolution: Late Quaternary shoreline morphodynamics, Cambridge: Cambridge University Press, p. 33-86, 1994.

DANIEL, H. Replenishment versus retreat: the cost of maintaining Delaware's beaches. Ocean \& Coastal Management. n. 44, p. 84-104, 2001.

DOMINGUEZ, J.M.L.; BITTENCOURT, A.C.S. P.; LEÃO, Z.M.A.N.; AZEVEDO, A.E.G. Geologia do quaternário costeiro do Estado de Pernambuco. Revista Brasileira de Geociências, v. 20, p. 208-215, 1990.

DOODY, J.P. Coastal Conservation and Management: an ecological perspective. Kluwer Academic Publishers, 308 p. 2001.

EULIE, D.O.; WALSH, J.P.; CARBETT, D.R. High-resolution analysis of shoreline change and application of balloon-based aerial photography, Albemarle-Pamlico estuarine system, North Caroline, USA. Limnology and Oceanography: Methods, v, 11 p. 151-160, 2013.

FERNANDEZ, D.; ARNOTT R.D.; BAUER B.O.; WALKER, I.J.; OLLERHEAD, J. Evaluation of the optimal resolution for characterizing the effect of beach surface moisture derived from remote sensing on aeolian transport Irene. Journal of Coastal Research, v. 65, 2013.

GONÇALVES, R.M.; COELHO, L.D.S; KRUEGER, C.P.; HECK, B. Modelagem preditiva de linha de costa utilizando redes neurais artificiais. Boletim de Ciências Geodésicas, v. 16, p. 420-444, 2010.

GONÇALVES, R.M.; AWANGE, J.; KRUEGER, C.P.; HECK, B.; COELHO, L.S. A comparison between three short-term shoreline prediction models. Ocean \& Coastal Management, v. 69, p. 102-110, 2012.

GONÇALVES, R.M.; AWANGE, J.; KRUEGER, C.P. GNSS- based monitoring and mapping of shoreline position in support of planning and management of Matinhos/PR (Brazil). Journal of Global Positioning Systems, v. 11, p. 156-168, 2012.

GONÇALVES, R.M.; PACHECO, A.D.P; TANAJURA, E.L.X.; DA SILVA, L.M. Urbanização costeira e sombreamento na praia de Boa Viagem, Recife-PE, Brasil. Revista de Geografia Norte Grande, v. 1, p. 241-255, 2013.

GUERRA, N.C \& MANSO, V.A.V. Beachrocks (Recifes de Arenito). In: ESKINAZI-LEÇAE. (Orgs.), Oceanografia: um cenário tropical. Recife, Bagaço, p. 109-130, 2004

HANTSON, W.; KOOISTRA L.; SLIM, P.A. Mapping invasive woody species in coastal dunes in the Netherlands: a remote sensing approach using LIDAR and high-resolution aerial photographs. Applied Vegetation Science. International Association for Vegetation Science, p.1-12, 2012

HAPKE, C.J.; REID, D.; RICHMOND, B.M.; RUGGIERO, P.; LIST, J. National assessment of shoreline change: Part 3: Historical shoreline change and associated coastal land loss along sandy shoreline of the California Coast. U.S. Geological Survey, Reston, p. 79, 2006.

HAPKE, C. \& PLANT, N. Predicting coastal cliff erosion using a Bayesian probabilistic model. Journal of Marine Geology, v. 278, p. 140-149, 2010.

HARVEY, A.M.; SILVA, P.G.; MATHER, A.E.; GOY, J.L.; STOKES, M.; ZAZO, C. The impact of Quaternary sea-level and climatic change on coastal alluvial fans in the Cabo de Gata ranges, southeast Spain. Geomorphology, v. 28, p. 1-22, 1999.

IBGE. www.ibge.gov.br/censo2010. Acesso, 2016.

MAGINI, C.; HARARI, J.; ABESSA, D.M.S. Circulação recente de sedimentos costeiros nas praias de santos durante eventos de tempestades: dados para a gestão de impactos físicos costeiros. Revista Brasileira de Geociências, v. 26, n. 4, p. 349-355, 2007.

MALLMAN, D.L.B. \& ARAÚJO, T.C.M. Vulnerabilidade do Litoral Sul de Pernambuco à erosão. Tropical Oceanography, v. 38, n. 2, p. 129-151, 2010.

MALLMAN, D.L.B.; PEREIRA, P.S.; NOGUEIRA, P.F.R.S. M.; SANTOS, F.M.M. Classificação morfodinâmica das praias arenosas de Ipojuca (Pernambuco, Brasil) através da análise semântica de imagens de satélite pancromáticas. Pesquisas em Geociências, v. 41, p. 169-189, 2014.

MADRUGA FILHO, J.D. \& ARAÚJO, T.C.M. Vulnerabilidade da zona costeira da praia do Paiva, Município do Cabo de Santo Agostinho, Pernambuco, Brasil. Tropical Oceanography, v. 28, n. 1, p. 1-10, 2000

MANSO, V.A.V.; ARAÚJO, T.M.; GUERRA, N.G.; SOARES JR, C.F.A. Pernambuco. In: DIETER, M. (Org.). Erosão e progradação do litoral brasileiro. 2. ed. Brasília, DF: Ministério do Meio Ambiente - MMA. p. 179-196, 2006.

MARTINS, K.A. \& PEREIRA, P.S. Coastal erosion at Pau Amarelo beach, northeast of Brazil. Journal of Coastal Research, v. 71, n. SI, p. 17-23,2014.

MAZZER, A.M.; DILLENBURG, S.R.; SOUZA, C.R.G. Proposta de método para análise de vulnerabilidade à erosão costeira no sudeste da ilha de Santa Catarina, Brasil. Revista Brasileira de Geociências, v. 38, n. 2, p. 278-294, 2008.

MENDONÇA, F.J.B.; GONÇALVES, R.M.; AWANGE, J.; SILVA, L.M.; GREGORIO, M.N. Temporal shoreline series 
analysis using GNSS. Boletim de Ciências Geodésicas, v. 20, p. 701-719, 2014.

MENEZES, J.T. Balanço de Sedimento Arenosos da Enseada do Balneário Camboriú-SC. Porto Alegre, 2008. 149p. Tese (Doutorado em Geociências), Universidade Federal do Rio Grande do Sul, Porto Alegre.

MORTON, R.A.; MILLER, T.A.; MOORE, L.J. National assessment of shoreline change: part 1: historical shoreline changes and associated coastal land loss along the U.S. Gulf of Mexico. U.S. Geological Survey, Reston, VA, p. 42, 2004.

MUEHE, D. Critérios morfodinâmicos para o estabelecimento de limites da orla costeira para fins de gerenciamento. Revista Brasileira de Geomorfologia, v. 2, n. 1, p. 35-44, 2001

NARDEZ, N.N.; GONÇALVES, RODRIGO, M.; SOARES, C. R.; KRUEGER, C.P. Classificação fuzzy da vulnerabilidade aos processos costeiros em Pontal do Paraná, Brasil. Pesquisas em Geociências, v. 43, p. 169-182, 2016.

NASCIMENTO, D.M.C. \& DOMINGUEZ, J.M.L. Avaliação da vulnerabilidade ambiental como instrumento de gestão costeira nos municípios de Belmonte e Canavieiras, Bahia. Revista Brasileira de Geociências, v. 39, p. 395-408, 2009.

NEVES, C.F. \& MUEHE, D. Vulnerabilidade impactos e adaptações às mudanças do clima: a zona costeira. In: Parcerias Estratégicas - mudança do clima do Brasil: vulnerabilidade, impactos e adaptação, n. 27, Brasília: Ministério da Ciência e Tecnologia, 2008.

PEREIRA, P.S.; CALLIARI, L.J.; HOLMAN, R.; HOLLAND, K.T.; GUEDES, R.M.C.; AMORIN, C.K.; CAVALCANTTI, P.G. Video and field observations of wave attenuation in a muddy surf zone. Marine Geology, v. 279, p. 210-221, 2011.

PEREIRA, P.S.; CALLIARI, L.J.; GUEDES, R.M.C. Variabilidade temporal dos bancos arenosos da praia do Cassino (RS): uma análise através de imagens de vídeo. Pesquisas em Geociências, v. 39, p. 195-211, 2012.

RIBEIRO, J.S.; SOUSA, P.H.G.O.; VIEIRA, D.R.; SIEGLE, E. Evolução da vulnerabilidade à erosão costeira na Praia de Massaguaçú (SP), Brasil Revista da Gestão Costeira
Integrada, São Paulo, v. 13 n. 3 p. 253-265, 2013.

RAPOSEIRO, P.D. \& FERREIRA, J.C.R. A análise da vulnerabilidade e do risco de inundação como ferramenta de apoio à gestão dos territórios litorais sob pressão urbana. Pluris: Planejamento urbano regional integrado e sustentável, p. 1-12, 2010.

RUDORFF, F.M. \& BONETTI, J. Avaliação da suscetibilidade à erosão costeira de praias da ilha de Santa Catarina. Brazilian Journal of Aquatic Science and Technology, v. 14, n. 1, p. 920, 2010.

SACRAMENTO, A.C.; CARMEN, S.; BEZERRA DE ALMEIDA, E. Aspectos florísticos da vegetação de restinga do litoral de Pernambuco. Revista Árvore, v. 31, n. 6. p. 11211130. 2007

SILVA, L.M.; GONÇALVES, R.M.; LIRA, M.M.; PEREIRA, P.S. Modelagem Fuzzy aplicada na detecção da vulnerabilidade à erosão costeira. Boletim de Ciências Geodésicas, v. 19, n. 4, p. 746-764, 2013.

SOUSA, P.H.G.O.; SIEGLE, E.T.M.G.; TESSLER, M.G. Environmental and Anthropogenic Indicators for Coastal Risk Assessment at Massaguaçú Beach (SP) Brazil. Journal of Coastal Research, v. 64, p. 319-323, 2011.

SOUSA, P.H.G.O.; SIEGLE, E.T.M.G.; TESSLER, M.G. Vulnerability assessment of Massaguaçú Beach (SE Brazil). Ocean \& Coastal Management, v. 77, p. 24-30, 2013.

TANAJURA, E.L.X.; KRUEGER, C.P; GONÇALVES, R.M. Análise da acurácia dos métodos cinemáticos de posicionamento GPS em aplicações costeiras. Boletim de Ciências Geodésicas, v. 17, p. 23-36, 2011

Submetido em 20 de setembro de 2016 Aceito em 9 de janeiro de 2018 